\title{
Explosive Nucleosynthesis: Prospects
}

\author{
David Arnett ${ }^{\mathrm{a}, 1}$, \\ a Steward Observatory, University of Arizona, Tucson AZ 85721, USA
}

\begin{abstract}
Explosive nucleosynthesis is a combination of the nuclear physics of thermonuclear reactions, and the hydrodynamics of the plasma in which the reactions occur. It depends upon the initial conditions - the stellar evolution up to the explosive instability, and the nature of the explosion mechanism.

Some key issues for explosive nucleosynthesis are the interaction of burning with hydrodynamics, the degree of microscopic mixing in convective zones, and the breaking of spherical symmetry by convection and rotation. Recent experiments on high intensity lasers provides new opportunities for laboratory testing of astrophysical hydrodynamic codes. Implications of supernovae 1987A and 1998bw (GRB980425?), and $\eta$ Carina are discussed, as well as the formation of black holes or neutron stars.
\end{abstract}

Key words: Nucleosynthesis, Hydrodynamics, Supernovae, Lasers, Black Holes, GRB's, Rotation, Convection, Jets

\section{Introduction}

This paper is presented to honor David Norman Schramm. It will be a personal view of where this field is going, not a review of work already done, and in a sense it is a formal continuation of many previous discussions Dave and I had on this topic.

There is a growing consensus that the synthesis of the elements and their isotopes may be divided into three major components:

- Cosmological synthesis of the light elements,

- Hydrostatic synthesis in stars, and

- Hydrodynamic synthesis in stellar explosions.

1 E-mail: darnett@as.arizona.edu 
The last item, "explosive nucleosynthesis," will be the focus here. Although thermonuclear burning has a morphological equivalent in terrestrial burning by chemical reactions, this discussion will be couched in the language of physics, and named dimensionless numbers, whose meaning may not be obvious to scientists outside the combustion and/or the fluid dynamics community, will be used sparingly. These numbers can be constructed from ratios of the time scales and of length scales discussed below.

Any discussion of nucleosynthesis requires consideration of the empirical basis of the nuclear reaction rates. The historical context and references are collected in [4]. The recent appearance of an excellent review [27] allows us the freedom here to concentrate instead upon topics less discussed but of not less importance: the hydrodynamic context of the burning. However, the brevity of this mention should not be interpreted as an indication that the construction of an empirical basis for nucleosynthesis theory is a finished topic. To use nucleosynthesis products as a probe of stellar environments, it is necessary to insure that abundance features observed and simulated are due to the history of physical conditions, not poorly known reaction rates. Nuclear physics is the foundation upon which we build.

Explosive nucleosynthesis is a combination of the nuclear physics of thermonuclear reactions, and the hydrodynamics of the plasma in which the reactions occur. It depends upon the initial conditions - the stellar evolution up to the explosive instability, and the nature of the explosion mechanism.

\section{Relevant Scales of Length and Time}

There is a vast difference between macroscopic and microscopic lengths in stars (see [4], Chapter 11). Burning is a nuclear process which occurs in a context of enormous dimension. The radius of the a typical star, the Sun, is $7 \times 10^{10} \mathrm{~cm}$, while the internuclear spacing is roughly $1.2 \times 10^{-8} \mathrm{~cm}(A / \rho)^{1 / 3}$, where $\rho$ is the density in cgs units and $A$ the mass number of the most abundant nucleus. The mean density of the Sun is $1.4 \mathrm{~g} / \mathrm{cc}$ (but in a presupernova densities increase to above $10^{9} \mathrm{~g} / \mathrm{cc}$ ). Suppose that collision cross sections have a scale of order $10^{-16} \mathrm{~cm}^{2}$, corresponding to a collisional mean free path of

$$
\lambda_{\text {col }} \approx 1.6 \times 10^{-8} A / \rho \mathrm{cm} .
$$

A characteristic scale for electron-photon interactions is the Thomson cross section, corresponding to a mean free path of roughly

$$
\lambda_{\text {rad }} \approx 1.6 \mathrm{~A} / \rho \mathrm{cm}
$$


The precise determination of cross sections, properly averaged over the relevant distributions is complex, but the qualitative result is the same as given by these simple estimates. This suggests that thermal energy moves much more easily than composition, and that our terrestrial intuition with flames may be colored by the ease with which heat moves on the length scales with which we are familiar.

Consider a homogeneous sphere of radius $R$; its diffusion time is

$$
\tau_{d i f}=\frac{3}{\pi^{2}} R^{2} / \lambda v=\frac{3}{\pi^{2}} R^{2} \rho N_{A} \sigma / v
$$

where $v$ is the velocity of the diffusing entities, $\sigma$ is the cross section, and $N_{A}$ is Avagadro's number. Diffusion is slow for large objects like stars. However, time scales for binary interactions are

$$
\tau_{\text {reac }} \approx 1 /\left(\rho N_{A} \sigma v\right)
$$

This dramatically different density dependence makes reactions faster and diffusion slower at higher densities, that is, at advanced burning stages.

Simulations of stellar hydrodynamics and evolution presume that the stellar plasma is homogeneous on scales below the size of the computational zone. However, this is unlikely for the late stages of stellar life, which occur on time scales of seconds, not billions of years. This scaling with density insures that the problem of incomplete mixing gets worse as the star evolves. It is exacerbated in massive stars because they evolve most rapidly; they are primary sites of explosive nucleosynthesis.

\section{Types of Nuclear Burning in Stars}

The simplest sort of stellar burning is that of a "radiative zone," in which heat can diffuse in and out, but composition is only changed by the conversion of fuel to ashes. This is thought to be the case in the center of the sun, for example, and the driving change which causes such stars to become red giants.

Even quasistatic nuclear burning can give locally intense heating, so that steep temperature gradients are formed which may drive convection. Convective currents are likely to be turbulent in stars because their large size and small viscosity give a large Reynolds number, that is, chaotic flow. This turbulent mixing is more effective than diffusion. As we shall see, the simulation of this represents a major challenge. Although such burning often is not explosive, it sets the stage for the explosive events, and seems to be their direct predecessor. 
A violent but relatively simple process is detonation [30]. The process is explosive, and supersonic. It is local in the sense that shock compression heats the fuel to the flash point, so that it incinerates. The motion of the shock is purely hydrodynamic except that the shock is enhanced by the energy released by the burning. The difficulty lies in determining how the detonation begins; this is an old and complex problem [18].

Another violent type of burning is deflagration [30], in which new fuel is ignited by heat flow from regions already burned. Unlike detonation, this depends upon the nature of the heat flow (conduction or radiative diffusion). Deflagration is subsonic, and in that sense is milder than detonation, into which it may develop. Deflagration is often unstable in stars (see Fig. 11.1 in [4]), which adds to the complexity of an already complex situation.

These themes, sufficiently complicated in their own right, are the basis for explosive nucleosynthesis. This level of complexity, and the need for quantitative predictions, makes computer simulations a necessary tool.

\section{Laboratory Supernova}

Before trusting hydrodynamic simulations, detailed quantitative testing of the computational framework is necessary. High intensity lasers have been successfully used to study the Rayleigh-Taylor (RT) and Richtmeyer-Meshkov (RM) instabilities well into the nonlinear regime [43]. The RT instability occurs when gravity tries to pull a heavier fluid through an underlying lighter one. The RM instability is similar except that the role of gravity is replaced by the inertial acceleration from the passage of a shock wave. Core collapse supernovae are driven by a poweful shock, and such shocks are the breeding ground of hydrodynamic instabilities. Observations of SN 1987A strongly suggested the occurrence of mixing of radioactive material outward, a phenomena not seen in one dimensional (1D) simulations but predicted in 2D simulations [5].

It is prudent to compare astrophysical codes to those mature computer codes used by the inertial confinement fusion (ICF) community on problems for which both should be applicable. This has been successfully done [26]; both CALE (ICF) and PROMETHEUS (astrophysics) codes were used to simulate an experiment on the NOVA laser at Lawrence Livermore National Laboratory (LLNL). The observed instabilities (RT and RM) were well simulated by both codes; the bubble and spike positions were reproduced, even into the strongly nonlinear regime.

A theoretical look at the relation between the hydrodynamics occurring in SN 1987A and in the laser experiments shows that a rigorous mapping exists 
[48]. Consider the He-H interface in the $\mathrm{SN}$ at $2000 \mathrm{~s}$, and the $\mathrm{Cu}-\mathrm{CH}$ interface in the laser experiment at $20 \mathrm{~ns}$. In both, the ratio of inertial to viscous forces (the Reynolds number) and the ratio of the convective to conductive heat transport (the Peclet number) are large. Therefore the viscosity and thermal diffusivity are negligible, and the dynamics of the interface well described by Euler's equations. These equations are invariant under a scale transformation, which maps lengths of $10^{11} \mathrm{~cm}$ into $50 \mu \mathrm{m}$, densities of $8 \times 10^{-3} \mathrm{~g} \mathrm{~cm}^{-3}$ into $4 \mathrm{~g} \mathrm{~cm}^{-3}$, and pressures of $40 \mathrm{Mbar}$ into $0.6 \mathrm{Mbar}$, for example, at a star time of $2000 \mathrm{~s}$ and a laser time of 20 nanoseconds. Thus, in a very real sense, the experiment reconstructed a part of the supernova event.

Such experiments are also crucial for another problem: multidimensional geometry. The experiments are inherently $3 \mathrm{D}$, but can be configured to give primarily $2 \mathrm{D}$ behavior. The computational load for $3 \mathrm{D}$ scales from $2 \mathrm{D}$ as the number of zones in the new dimension. At present, a single workstation can easily produce $2 \mathrm{D}$ simulatons with good resolution, but $3 \mathrm{D}$ requires parallel processing. For the next few years we will need to explore with $2 \mathrm{D}$ while we develop the capability of doing reliable and resolved 3D simulations with ease. The laser experiments can help us discover the qualitative and quantitative limitations of $2 \mathrm{D}$ in real world situations.

Laboratory experiments can have other impacts on explosive nucleosynthesis. Experiments modelling turbulent mixing, combustion, and flame propogation are needed. However, because the scales of the systems are so different, care must be taken in mapping the experiments into the astrophysical domain.

\section{Thermonuclear Supernovae}

Supernovae of Type Ia are thought to be produced in a white dwarf star by a runaway thermonuclear reaction. They produce radioactive ${ }^{56} \mathrm{Ni}$, and its decay to ${ }^{56} \mathrm{Co}$ and thence to ${ }^{56} \mathrm{Fe}$ give the characteristic light curve [4]. SNIa's are a major source of ${ }^{56} \mathrm{Fe}$ and other iron-group nuclei. Because of the empirical relationship between their brightness and the width of the luminosity peak (the Phillips relation), they are the best distance indicators now known, and of fundamental importance for cosmology $[41,44,40]$.

Supernovae of Type Ia present several outstanding puzzles.

- What are their progenitors?

- How do they evolve to ignition?

- How do they evolve from ignition to explosion?

- Why does the Phillips relation between brightness and peak width work? 
At present there is no unique and satisfactory scenario for their evolution up to explosion. For example, SNIa's may result from one member of a binary pair growing in mass from accretion by its companion (there are many possibilities for the nature of that companion and of the matter accreted), or by the merger of a pair of white dwarfs (which is an inherently 3D problem, and not yet computable for secular time scales and good resolution).

If the accretion model is the correct one, how does it ignite? The favorite notion is that it ignites ${ }^{12} \mathrm{C}$ at the core of the white dwarf. To avoid collapse or excessive production of neutron rich isotopes, the ignition must not occur at densities which are too high $\left(\rho>>2 \times 10^{9} \mathrm{~g} / \mathrm{cc}\right)$. From ignition to thermal runaway there is a period of about $10^{3}$ years, during which a convective region with Urca cooling probably evolves ( see references and discussion in [4,37]), but such a process has only been simulated in 1D. What occurs in this "lost millenium" remains puzzling. See also [45] for simulations of the closely related problem of the core evolution of a $11 M_{\odot}$ star.

If the burning does proceed to thermal runaway, the following evolution remains a subject of debate $[39,54,28]$. It may take a new generation of simulations to resolve the issue.

The merger scenario also has uncertainty regarding the cause of explosion. Many discussions ( e.g., [23]) assume that given a combined mass above the chandrasekhar limit, explosion must ensue. This is untrue. Collapse or benign ignition are also possible, maybe even more likely $[38,49]$. Pioneering efforts to simulate the merger process probably need better resolution and longer evolutionary times to get at this problem, or the related one of mergers involving other combinations of constituents (white dwarfs, neutron stars, black holes) $[15,50,42,46,47]$.

Presumably from all this confusion will emerge a natural and convincing reason for the Phillips relation.

\section{Almost Explosive Burning — Setting the Stage}

Consider an evolved massive star, nearing core collapse. Its oxygen burning shell is an important region for explosive nucleosynthesis: this layer is the site of explosive oxygen and silicon burning as it is ejected by the supernova shock. Its formation and development set the stage for the collapse of the burned core to form a neutron star or black hole. Any discussion of core collapse, explosion mechanisms, or continued collapse to a black hole must presume characteristics of this formation and development, an issue we will return to below. 
Almost all simulations of the stages prior to core collapse have been one dimensional (1D), assuming spherical symmetry and instantaneous mixing of radial layers over all angles. In $2 \mathrm{D}$ it is possible to treat convection as a real hydrodynamic process, although the vortices are pegged to the grid by assumption. Such simulations $[7,6]$ are qualitatively different from the previous $1 \mathrm{D}$ ones.

This should not be surprising. The time scales are very short for such a large object. The duration of shell oxygen burning is only $10^{4}$ seconds or so, the connective turnover time about $10^{2}$ seconds, and the sound travel time about 1 second. The sound speed is about 0.01 of lightspeed. There is little time for the convection or the burning to settle into a steady state, or to make the zone well mixed in composition (or even in heat), contrary to the assumptions built into the stellar evolutionary codes. Strong downdrafts develop, and the convection is nonlinear and nonsymmetric with regard to up and down flows. The convective mach numbers approach tenths, and the perturbations in pressure and density are of the order of tens of percent at the flame zone and at the interface at the top of the formally convective zone. The burning is sporadic and flashy. Perhaps the most erroneous aspect of the 1D codes is their treatment of the boundary conditions on convection. Material moves across formally stable regions; in the $2 \mathrm{D}$ simulations ${ }^{12} \mathrm{C}$ was entrained from across the outer interface of the oxygen convective zone, and brought down into the flame zone where it flashed vigorously. This occurred after about 400 seconds in the Bazan-Arnett computation [7].

These results have now been confirmed and extended by a completely independent hydrodynamic code and method [6]. At 900 seconds there seems to be a new state developing, strongly dynamic but roughly steady on average. The nuclear luminosity increased more than a factor of 30 above the value obtained in the 1D simulations. The burning resembles a series of "mini-explosions", in which the burning happens at higher temperatures in flashes, separated by relatively quiet intervals.

The most obvious conclusion is that the 1D simulations of the final stages of massive stars are unrealistic, and their degree of relevance is in question, at least regarding details of presupernovae and pre-explosion nucleosynthesis. We can already see that the shell luminosities are incorrect, as are the mixing algorithms. This brings into question the pre-collapse states hitherto used for core collapse simulations. These simulations show a dependence upon the neutronization (that is, upon $Y_{e}$ ), the mass of the burned core, and its entropy. All these features may change.

While these new simulations do provide a first hydrodynamic description of convection for this evolutionary stage, they must be improved. First, they should be pushed all the way to core collapse, in order to determine the quan- 
titative extent of the changes. Second, their 2D nature may be suspect. Rotation and magnetic fields, unavoidable is this stellar plasma, may reduce the geometric complexity, tending back toward 2D. On the other hand, vortex wandering in $3 \mathrm{D}$ may reduce the effects seen in $2 \mathrm{D}$. This challenge is becoming tractable with progress in computer hardware and software.

It may be that the notion, that the extent of the burning shell is determined by the local adiabatic gradient, is flawed. The convective velocity field is certainly NOT local. Rather, the depth to which a blob sinks depends upon which fuels it has to flash and reverse its descent, upon how low its entropy drops due to previous neutrino cooling, and upon its history of electron capture. The actual compositional structure may be better thought of as an ensemble average of blobs being subjected to these effects. This view would imply revisions of hydrostatic as well and explosive nucleosynthesis yields, at least in detail, and perhaps in general.

In this picture, an important argument against significant rotation is removed. The abundances in a rapidly stirred region would be representative of the flame zone in that region, so that rotational mixing would tend to destroy the compositional layering needed to reproduce the solar system abundance pattern. However, if blobs were self limiting in their motion, depending upon their composition, the layering would represent both the temperature and compositon, and could survive.

\section{Core Collapse Supernovae}

The dramatic rotational symmetry of the rings of SN1987A, and of the eruption of $\eta$ Carina, suggest that rotation is important for at least the late stages of evolution of massive stars. Given this hint, let us re-examine how rotation might and might not have consequences for supernovae. It appears that one of the worst problems, the destruction of compositonal layering just discussed, may be moot with the new view of convective burning in the presupernova.

\subsection{The Neutrino Diffusion Model}

In the Colgate model of core collapse [13], the collapsing core was supposed to be both thick and thin to neutrinos. It had to be thick so that neutrinos were copiously produced, but thin so they would stream out, but thick enough again to deposit energy as they escaped. The neutrino transport was not actually calculated in [13], but assumed to work in this fashion. That is, the neutrinos were assumed to diffuse quickly out of the collapsed core, and half of their 
energy was deposited in the mantle. All the models so calculated gave violent explosions; no black holes were formed.

The first radiation hydrodynamic calculations $[1,2]$ showed that if such fine tuning were allowed, explosions did result, but also showed that black hole formation with no explosion was also likely. This follows from a simple argument. The diffusion time out of a homogeneous sphere of mass $M$ is

$$
\tau_{\text {dif }}=\frac{3}{\pi^{2} c} \kappa(3 M / 4 \pi)^{2 / 3} \rho^{1 / 3}
$$

where $\kappa$ is the neutrino opacity and $\rho$ the density. The collapse becomes supersonic, so that it takes a time which is of the order of and scales with the free fall time, $\tau_{f f} \propto R / v_{f f}$. Since $v_{f f}^{2}=2 G M / R$, we have $\tau_{c} \propto \rho^{-1 / 2}$. The degree of neutrino trapping depends upon the ratio of diffusion time to collapse time,

$$
\tau_{d i f} / \tau_{c} \propto \kappa \rho^{5 / 6} M^{2 / 3}
$$

Increasing the neutrino opacity, the density, or the core mass $M$ tends to increase the neutrino trapping, and reduce the chance of explosion. Massive cores tended to make black holes. With the advent of the neutral current theory of weak interactions, the effective value of the neutrino opacity increased, increasing trapping. Modifying the inital models or the nuclear equation of state to give higher density at bounce also increased trapping. If the neutrinos are trapped in the core, the collapse continues on to black hole formation. Nor does arbitrary tuning of $\kappa$ fix things. If the neutrino opacity is low, the neutrinos escape but do little heating of the surrounding and infalling mantle.

These parameters are not freely variable. The mass $M$ is constrained by the progenitor core mass, which itself cannot be less than the chandrasekhar mass. The weak interaction determines $\kappa$ fairly precisely. The density of the core has varied in simulations, but more care, realistic evaluation of nuclear equation of state, and inclusion of general relativity, give more tightly constrained values. It seems fair to say that the neutrino diffusion model does not work with realistic physics. See [34] for a recent review of the status of core collapse models.

\subsection{Energetics}

The unusual supernova 1998bw, and its possible identification [19,24,53] with the gamma-ray burst GRB980425, suggest that the supernova mechanism should be able to provide large explosion energies $\left(E_{\text {exp }} \geq 10^{52} \mathrm{erg}\right.$, or more 
than 10 foe). The gravitational potential energy is

$$
\Omega \approx G(4 \pi / 3)^{1 / 3} M^{5 / 3} \rho^{1 / 3}
$$

in the newtonian approximation. The minimum core density is about that of the atomic nucleus,

$$
\rho_{\text {nuc }}=2 \times 10^{14} \mathrm{~g} / \mathrm{cc}
$$

A schwarzschild black hole has an average density of

$$
\rho_{b h}=3 c^{6} / 32 \pi G^{3} M^{2}=2.85 \times 10^{16}\left(M_{\odot} / M\right)^{2} \mathrm{~g} / \mathrm{cc},
$$

but bounce densities as low as $10^{15} \mathrm{~g} / \mathrm{cc}$, dynamical formation of a black hole can occur. Thus $2 \times 10^{14} \leq \rho \leq 10^{15}$ gives an estimate for the maximum energy available for explosion. In the spherically symmetric case, black hole formation at higher masses will limit the energy available for explosion; just below this boundary the energy supply is at a maximum, e.g. [52].

\subsection{The Shock Mechanism}

If diffusion of neutrinos will not give adequate transport of energy, shock propagation might; Bethe and Brown have led the pursuit of this possibility [8]. Upon reaching nuclear density, or higher, the pressure is adequate to support the collapsing core. The size of this region is given by equating the pressure gradient force to gravity. The mass, for which this is true, falls as a unit, with velocity proportional to radius, so it is called the "homologous core." Prior to collapse the central density reaches $\rho \geq 2 \times 10^{9} \mathrm{~g} / \mathrm{cc}$. Neutrino cooling keeps the entropy low, so that the "iron core mass" approaches the chandrasekhar value,

$$
M_{c h} / M_{\odot} \approx 1.45\left(2 Y_{e}\right)^{2}
$$

where $Y_{e}$ is the number of electrons per nucleon. The electron fermi energy is several $\mathrm{MeV}$. Electron capture occurs relatively slowly, but at $Z / A=Y_{e} \approx$ 0.42 , the nuclei have a threshold for electron capture of several Mev as well, and the neutronization is almost stopped [4]. The smallest iron core is about $1.0 M_{\odot}$ for $y_{e}=0.42$. The largest homologous core would occur if no leptons were lost in subsequent collapse, so their pressure would have the largest possible value. At nuclear density, the difference between proton and neutron 
chemical potentials will be small compared to the fermi energies of electrons and neutrinos (of order $100 \mathrm{Mev}$ ), so at weak-interaction equilibrium,

$$
\mu_{e} \approx \mu_{\nu}
$$

This implies $N_{e} / g_{e}=N_{\nu} / g_{\nu}$, where the helicity phase space factors are $g_{e}=2$ and $g_{\nu}=1$, so $Y_{e}=2 Y_{\nu}$, and with no lepton escape we have for the collapsed core $Y_{e}=0.28$ and $Y_{\nu}=0.14$. The pressure defict is $P / P_{0}=(2 / 3)^{4 / 3}+$ $\frac{1}{2}(1 / 3)^{4 / 3} \approx 0.700$. The corresponding mass deficit is $(0.7)^{3 / 2}=0.585$, so that only about $60 \%$ of the iron core is still in the homologous core when it reaches nuclear density and bounces. The shock must propagate through about $40 \%$ of that iron core which is still infalling. For the shock to be strong, it must dissociate the iron, requiring $8 \mathrm{MeV}$ per nucleon, or 6.4 foe $\left(6.4 \times 10^{51} \mathrm{erg}\right)$. The energies available in such small collapsing cores is almost always smaller than this, thus making the shock mechanism doubtful.

\subsection{The Convective Mechanism}

Although the core collapse releases much more energy than seems to be necessary for the typical supernova, the problem lies in getting it out of the nascent neutron star/black hole. One possible solution is "convective overturn." The term "overturn" is important because the process is unlikely to resemble a well-developed turbulent cascade, but rather a more violent and transient large scale turnover of the lepton-rich deep regions. Epstein [16] first examined the consequences of this possibility. Early numerical simulations [11,32] and arguments [14] based on this idea were shown by Smarr et al. [51] to be overly enthusiastic, although the overturn of the outer core would be a generic and important feature of the core collapse.

Given the difficulties of the neutrino diffusion and the shock models, it would appear better to allow almost all of the iron core to fall in, then release the neutrinos at a later time. Because there would be less mass to dissociate, this minimizes the dissociation losses. The first simulation which showed such "delayed" behavior is due to Wilson [9]. This was a 1D simulation and therefore had a dubious treatment of convective flow. It did stimulate multidimensional simulations $[21,12,25,35]$ which gave results still being argued.

\subsection{The First Rotational Mechanism}

Fred Hoyle $[22,17]$ proposed that rotation played an important role in supernovae. If there is enough angular momentum in the oxygen shell layer which 
surrounds the iron core, collapse might induce explosion by oxygen burning. This did not result in the Colgate-White simulations because their collapse generated an excessively strong rarefaction wave which swallowed the oxygen shell. A more careful treatment of the onset of core collapse [3] showed a longer initial contraction, in which the oxygen shell did burn violently (but not quite explosively). Following Fowler and Hoyle's suggestion [17], Bodenheimer and Woosley [10] simulated some simple explosions of this type, with rough estimates of the rotational state of the presupernova. These should be re-examined using more realistic multidimensional precollapse models and better resolution. The combined effects of rotation and hydrodynamics on the core mass, the neutron excess, and the entropy would be interesting in its own right. Even in the 1D case the explosion of SN1987A would have gotten about $10 \%$ of its energy from explosive burning of oxygen.

\subsection{Rotation Revisited}

Rotation could cause significant effects in the core itself [36]. Extreme rotation would cause the collapse to halt due to centrifugal forces at a density less than nuclear density. While this would aid neutrino escape, it would also release less gravitational energy and lower the neutrino energies, making them less able to deposit energy in the outer layers. An unresolved issue is the rate of angular momentum transport, which in this case would determine the secular evolution to the neutron star or black hole state.

Magnetic fields might be important as well $[22,20,31]$. The plasma is matter, not field, dominated (a high $\beta$ plasma), so that magnetic fields would be subtle, at least initially. With dynamo action the field would be strengthened, and buoyancy would tend to move it to regions in which its effects might be still more important. This is justifiably an old problem because it is inherently 3D and time dependent. The failure of the pulsar models to predict a luminosity below the observed radioactive decay of ${ }^{56}$ Co in SN1987A may indicate that magnetic effects are not a dominant feature; however evidence for a pulsar (or alternatively a black hole) would clarify this point.

A more modest and perhaps realistic (?) role for rotation is to induce mixing, but not centrifugal braking. The rotation and magnetic field would guide the overturn, emphasizing large scale motion (low modes). This may help the "convection" model discussed above.

In any case, rotation is likely to have an important effect in that it breaks the spherical symmetry in a characteristic way. While neutrino diffusion of energy will tend to be spherically symmetric, rotation tends to give the rotation axis a special role. Centrifugal force tends to evacuate these regions, so that 
they would have a lower density and, if heat transport is effective, a higher entropy. Such polar hot spots might be conducive to the formation of jets, and trigger overturn. Preliminary attempts to examine the consequences $[29,33]$ are promising.

\section{Summary}

- Explosive nucleosynthesis is a combination of nuclear reactions with hydrodynamics, and depends upon explosions mechanisms for supernovae.

- Incomplete mixing gets worse for massive stars and explosive conditions.

- Nuclear burning, and yield predictions, is complicated by hydrodynamic convection in presupernovae.

- Laboratory experiments with high intensity lasers has become a good testing ground for astrophysics codes. With careful scaling, such experiments can reproduce supernova phenomena.

- Understanding of SNIa's is impeded by a lack of progenitor information, and by theoretical problems with approach to ignition, runaway, and stellar merger.

- Simulations of oxygen shell burning using actual (2D) hydrodynamics differ drastically from 1D results. The first simulations have been confirmed by an independent code, and is being carried further.

- For core collapse explosion mechanisms, neutrino diffusion and prompt shock models are dead, and pure convection models may be sick.

- Rotation must be included in progenitor and core collapse evolution.

- SN1998bw shows that the energy problem with core collapse supernovae is worse than previously supposed. There are events having energies much larger than several foe $\left(10^{51} \mathrm{erg}\right)$.

- Because newtonian gravity and centrifugal force are scale free, jet formation is likely to occur on scales having effective heat flow. This may connect protostar jets and galactic jets (with heat flow by radiative diffusion and convection), and core collapse supernovae (with heat flow by neutrino diffusion and convection).

The prospects are simply wonderful. Our tools are getting much better, and may finally be up to the task of simulation of explosive nucleosynthesis events in realistic geometry. Computer technology, nuclear reaction rates, and hydrodynamics rates are improving and being verified in new ways. Meteoritic data [55] has presented quantitative challenges to 1D model predictions. With the impending crash of SN1987A into its rings, the renewed activity of $\eta$ Carina, the active supernova searches out to large redshift, and the possibility of a connection between core collapse supernovae and GRB's, we may expect to learn many new things. 


\section{Acknowledgment}

This research is supported by DOE grant DE-FG03-98DP00214/A001.

\section{References}

[1] D. Arnett, 1966, Can. J. Phys. 44, 2553

[2] D. Arnett, 1967, Can. J. Phys. 45, 1621

[3] D. Arnett, 1977, ApJ 218, 815

[4] D. Arnett, 1996, Supernovae and Nucleosynthesis, (Princeton University Press: Princeton NJ)

[5] D. Arnett, B. A. Fryxell, \& E. Müller, 1989, ApJ 341, L63

[6] S. Ashida, E. Livne, \& D. Arnett, 1999, in preparation

[7] G. Bazan \& D. Arnett, 1998, ApJ 496, 316

[8] H. A. Bethe, 1990, Rev. Mod. Phys 68, 801

[9] H. A. Bethe \& J. R. Wilson, 1985, ApJ 295, 14

[10] P. Bodenheimer, \& S. E. Woosley, 1983, ApJ 269, 381

[11] S. W. Bruenn, J. R. Buchler, \& M. Livio, 1979, ApJ 234, L183

[12] A. Burrows, J. Hayes, \& B. A. Fryxell, 1995, ApJ 450, 830

[13] S. A. Colgate \& R. H. White, 1966, ApJ 143, 626

[14] S. A. Colgate \& A. G. Petchek, 1980, ApJ 236, L115

[15] M. B. Davies, W. Benz, \& J. G. Hills, 1994, ApJ 424, 870

[16] R. I. Epstein, 1979, MNRAS 188, 305.

[17] W. A. Fowler \& F. Hoyle, 1964, ApJS 9, 201

[18] W. Fickett \& W. C. Davis, 1979, Detonation, (University of California Press: Berkeley CA)

[19] T. J. Galama, et al., 1998, Nature 395, 670

[20] J. E. Gunn, \& J. P. Ostriker, 1971, ApJ 160, 979

[21] M. E. Herant, W. Benz, W. R. Hix, C. Fryer, \& S. A. Colgate, 1994, ApJ 435, 339

[22] F. Hoyle, 1946, NMRAS 106. 343 
[23] I. Iben, Jr., \& A. V. Tutukov, 1999, ApJ 511, 324

[24] K. Iwamoto, et al., 1998, Nature 395, 672

[25] H.-Th. Janke, \& E. Müller, 1996, AAp 306, 167

[26] J. Kane, et al., 1997, ApJ 478, L75

[27] F. Käppler, F.-K. Thielemann, \& M. Wiescher, 1998, Annu. Rev. Nucl. Part. Sci. 48, 175

[28] A. Khokhlov, 1991, AAp 245, 114

[29] A. Khokhlov, et al., 1999, ApJ, submitted

[30] L. D. Landau \& E. M. Lifshitz, 1959, Fluid Mechanics, (Addison-Wesley: New York)

[31] J. M. LeBlanc \& J. R. Wilson, 1970, ApJ 161, 541

[32] M. Livio, J. R. Buchler, \& S. A. Colgate, 1980, ApJ 238, L139

[33] A. MacFadyen \& S. E. Woosley, 1999, astro-ph/9810274

[34] A. Mezzacappa \& S. W. Bruenn, 1998, in proceedings of Future Directions is Supernova Research, to appear in Memoirs of the Italian Astronomical Society, held at Assergi, Italy, Sept. 29-Oct. 2, 1998

[35] A. Mezzacappa, et al., 1997, ApJ 495, 911

[36] R. Mönchmeyer \& E. Müller, 1989, in NATO ASI on Timing Neutron Stars, ed. H. Ögelman \& E. van den Heuvel, (Dircrecht: Kluwer), p. 549

[37] R. Moschkovitch, 1996, AAp 311, 152

[38] R. Moschkovitch, \& M. Livio, 1990, AAp 236, 378

[39] J. Niemeyer \& W. Hillebrandt, 1995, ApJ 452, 779

[40] S. Perlmutter, et al., 1997, ApJ 517, 565

[41] M. M. Phillips, 1993, ApJ 413, L105

[42] F. Rasio, 1994, BAAS 184, 41.12

[43] B. A. Remington, et al., 1994, Phys. Plasmas 4, 1994

[44] A. Riess, W. H. Press, \& R. P. Kirshner, 1995, ApJ 438, L17

[45] C. Ritosso, E. Garcia-Berro, \& I. Iben, Jr., 1999, ApJ 515, 381

[46] M. Ruffert, 1997, in Proceedings of Fourth Huntsville Gamma-Ray Burst Symposium, 15-20 Sept., 1997

[47] M. Ruffert, \& H.-Th. Janke, 1999, AAp 344, 573

[48] D. Ryutov, et al., ApJ, in press (June, 1999) 
[49] H. Saio, \& K. Nomoto, 1999, ApJ 500, 388

[50] L. Sergretain, C. Chabrier, \& R. Mochkovitch, 1997, ApJ 481, 355

[51] L. Smarr, J. R. Wilson, R. T. Barton, \& R. L. Bowers, 1981, ApJ 246, 515

[52] K. A. van Riper, \& D. Arnett, 1979, ApJ 225, L129

[53] L. Wang \& J. C. Wheeler, 1998, ApJ 504, L87

[54] S. E. Woosley, 1994, in Supernovae, ed. S. A. Bludmann, R. Mochkovitch, J. Zinn-Justin, (Amsterdam: Elsevier Science)

[55] E. Zinner, 1998, Annu. Rev. Earth Planet. Sci. 26, 147 